\title{
NOTAS SOBRE EL CONTROL \\ DE CLÁUSULAS ABUSIVAS ENTRE EMPRESARIOS. UNA SÍNTESIS DEL MODELO ALEMÁN*
}

SOME REFLECTIONS

ABOUT UNFAIR TERMS BETWEEN BUSINESS.
A GERMAN MODEL SYNTHESIS

Pamela Mendoza Alonzo"*

María Elisa Morales Ortiz

\section{RESUMEN}

Sin propósitos comparativos y a modo de ensayo, este trabajo presenta un breve análisis de la normativa alemana sobre control de cláusulas abusivas en contratos entre empresarios. El trabajo, además, expone la interpretación jurisprudencial extensiva de protección a pequeños empresarios de los tribunales alemanes y concluye explicando la crítica a esta misma tendencia y enuncia las propuestas de reforma al respecto.

Palabras clave: $B G B$; cláusulas abusivas; derecho del consumo; B2B; derecho alemán.

\section{AbStRact}

No comparative purposes and as an essay, this paper presents a brief analysis of the German law on the control of unfair terms in contracts between business. In addition, it exposes the extensive interpretation of protection to small businessmen by the German courts and concludes explaining the criticism to this same trend and stating the reform proposals in this regard.

Keywords: $B G B$; Unfair terms; Consumer law; B2B; German law.

* Este trabajo es parte del proyecto Fondecyt Iniciación n. ${ }^{\circ} 11190543$ titulado "Criterios de verificación de asimetría en los contratos B2B. Una perspectiva de Derecho Comparado".

${ }^{* *}$ Profesora de Derecho Civil de la Facultad de Ciencias Jurídicas y Sociales, Universidad Austral de Chile. Dirección postal: Los Pinos S/N, Balneario Pelluco, Puerto Montt, Chile. Correo electrónico: pamela.mendoza@uach.cl.

*** Profesora de Derecho Civil y Derecho del Consumo de la Facultad de Ciencias Jurídicas y Sociales, Universidad Austral de Chile. Dirección postal: Avenida Rector Eduardo Morales Miranda, n. ${ }^{\circ}$ 23, Valdivia, Chile. Correo electrónico: maria.morales@uach.cl. Este trabajo se sometió a double blind review y fue recibido el 29 de noviembre de 2019 y aceptado para su publicación el 3 de marzo de 2020. 


\section{INTRODUCCIÓN}

En otro trabajo, de un análisis de la evolución de la regulación de las cláusulas abusivas en los ordenamientos inglés, alemán y francés ${ }^{1}$, se concluye que los "sistemas padres"2 coinciden en la extensión del control de cláusulas abusivas a relaciones entre empresarios o $\mathrm{B}_{2} \mathrm{~B}^{3}$, pero con distinta técnica legislativa. Esto último permite hablar de tres modelos diferentes en esta materia.

En cuanto al modelo alemán, es posible sostener que se ha preocupado de manera temprana de la regulación las relaciones asimétricas como categoría general, más que solo de las relaciones de consumo propiamente tal (B2C), pudiendo catalogarse como un sistema vanguardista en este sentido.

A continuación, a modo de ensayo, se realizará una breve síntesis de la normativa del control de cláusulas abusivas en contratos B2B en el sistema alemán, para luego explicar algunas de las críticas y propuestas de reforma que han surgido al respecto.

\section{SíNTESIS SOBRE LA REGULACIÓN DE LAS CLÁUSULAS ABUSIVAS ENTRE EMPRESARIOS EN EL DERECHO ALEMÁN ${ }^{4}$}

Desde una perspectiva comparativa, el derecho alemán ha sido pionero en la extensión del control de cláusulas abusivas a contratos entre empresarios. Así, la ley sobre condiciones generales de la contratación de 1976 (AGBG) resultaba aplicable tanto a las relaciones de consumo como a relaciones $\mathrm{B}^{2} \mathrm{~B}^{5}$.

Sin embargo, como explica Reinhard Zimmermann, la AGBG no se trataba en estricto rigor de una ley de protección al consumidor, sino de una regulación sobre los términos generales del contrato, estableciendo un catálogo de cláusulas abusivas, recogidas de una larga tradición jurisprudencial, aplicables cada vez que exista una parte débil en la relación contractual ${ }^{6}$. Dichos criterios jurisprudenciales sobre términos y condiciones generales se desarrollaron en un inicio en torno a la noción de buenas costumbres del apartado 1 del $\S 138^{7}$ del $B G B^{8}$, para posteriormente ampliarlo, a partir de la década de 1950, a los estándares de la buena fe contractual del $\S 242$ del $B G B^{9}$.

${ }^{1}$ Morales, Mendoza y Munita (2020).

${ }^{2}$ ZwEIgert \& KÖTZ (1998), p. 41, aconsejan la selección de estos sistemas.

${ }^{3}$ Preferimos esta última expresión.

${ }^{4}$ Una revisión comparativa se puede revisar en Morales, Mendoza y Munita (2020).

${ }^{5}$ Zimmermann (2005), p. 434.

${ }^{6}$ Op. cit., p. 431.

${ }^{7} \S 138$ Sittenwidriges Rechtsgeschäft; Wucher (1) Ein Rechtsgeschäft, das gegen die guten Sitten verstößt, ist nichtig. al $B G B$.

${ }^{8}$ En este apartado, cualquier referencia a un artículo sin especificación se entiende hecha

${ }^{9}$ Zimmermann (2005), p. 433. Véase jurisprudencia al respecto en Gsell (2017), p. 239, notas 8 y 9. § 242 Leistung nach Treu und Glauben Der Schuldner ist verpflichtet, die Leistung so zu bewirken, wie Treu und Glauben mit Rücksicht auf die Verkehrssitte es erfordern. 
Más adelante, con el fin de armonizar el derecho interno con la Directiva 93/13/CEE del Consejo, de 5 de abril de 1993, sobre las cláusulas abusivas en los contratos celebrados con consumidores, la AGBG fue reformada el 19 de julio de 1996 (Gesetz zur Änderung des AGB-Gesetzes und der Insolvenzordnung) ${ }^{10}$. En dicha reforma destaca la modificación del $\S 12$ (Internationaler Geltungsbereich) y un nuevo artículo, el § 24a (Verbraucherverträge), relativo a los "contratos de consumo", donde se fortaleció la protección al consumidor por sobre otros contratantes débiles ${ }^{11}$. Con todo, la AGBG siguió siendo una ley de condiciones generales de la contratación de aplicación general ${ }^{12}$.

Más tarde, con las reformas al $B G B$ del año 2000 (que incorporó los $\S 13$ 14) y de 2001 (que derogó la AGBG y la incorporó en los §§ 305-310) esta tendencia de protección amplia se mantuvo. Ambas reformas, terminaron de adaptar (aunque sin grandes modificaciones) el derecho nacional, en este tema, a la directiva 93/13/CEE.

En efecto, la AGBG fue derogada con la Ley de Modernización del Derecho de Obligaciones de 26 de noviembre de 2001 (Gesetz zur Modernisierung des Schuldrechts), que entró en vigor el 1 de enero de 2002. Así, fue integrada "en forma de bloque en el $B G B$ "13 bajo el título "Configuración de relaciones obligatorias negociales mediante condiciones generales de la contratación" ${ }^{14}$ (Gestaltung rechtsgeschäftlicher Schuldverhältnisse durch Allgemeine Geschäftsbedingungen) del libro 2, relativo al derecho de las obligaciones ${ }^{15}$. Así, hoy los $\S 305-310$ del $B G B$ son las normas referentes en esta materia. Esta especial configuración del Código Civil alemán representa, en la actualidad, un modelo de derecho del consumo ${ }^{16}$.

En ese orden de cosas, el $§ 305$ (Einbeziehung Allgemeiner Geschäftsbedingungen in den Vertrag) define lo que son las condiciones generales de la contratación y establece cuándo se entienden incorporadas al contrato ${ }^{17}$. Por su parte, el § 305a (Einbeziehung in besonderen Fällen) se refiere a la

${ }^{10}$ En todo caso, es sabido que la AGBG sirvió en parte de inspiración en la redacción de la misma directiva.

${ }^{11}$ Zimmermann (2005), p. 441.

${ }^{12}$ Albiez (2002), p. 1148.

${ }^{13}$ WACKe (2013), p. 705.

${ }^{14}$ Seguimos la traducción de Vives (2002), p. 1243.

${ }^{15}$ Decisión que no estuvo exenta de polémica, como explica Albiez (2002), p. 1147, "después del BGB, según muchos, la AGB-G ha sido el mejor logro de la cultura iusprivatista alemana ¿Por qué había que romper este edificio tan bien diseñado?”. Del mismo autor, véase listado de leyes especiales sobre derecho de consumo también incorporadas en otros artículos del $B G B$, pp. 1151-1553

${ }^{16}$ Para Rodrigo Momberg, junto al caso holandés, se trata de un modelo de vinculación normativa entre el derecho privado común y la normativa de protección a los consumidores. Momberg (2016), p. 739.

${ }^{17}$ En lo sucesivo seguiremos la traducción de los arts. $\S \S 305-310$ del $B G B$ de VIVES (2002), pp. 1245-1253. 
incorporación en casos especiales, el § 305b (Vorrang der Individualabrede) establece la prevalencia de los acuerdos individuales sobre las condiciones generales de la contratación, mientras que el § 305c (Überraschende und mehrdeutige Klauseln) se refiere a las cláusulas sorpresivas y ambiguas. El $\S 306$ (Rechtsfolgen bei Nichteinbeziehung und Unwirksamkeit) regula las consecuencias jurídicas en caso de no incorporación e ineficacia, en tanto el $\S 306$ a (Umgehungsverbot) impone la prohibición de elusión de la normativa mediante alguna otra configuración contractual.

Destaca, en especial ${ }^{18}$, el $\S 307$ (Inhaltskontrolle) que, consagrando una línea jurisprudencial, agrega un control de contenido disponiendo:

(1) Las cláusulas de las condiciones generales de la contratación contrarias a la buena fe son ineficaces si perjudican de forma indebida a la contraparte del predisponente. Un perjuicio indebido puede resultar también cuando la cláusula esté redactada de forma no clara e incomprensible ${ }^{19}$.

El apartado 2, por su parte, aclara cuáles son los casos en que se presume un "perjuicio indebido". Esta norma tiene un alcance general, por lo que es la norma fundamental del ámbito de protección del empresario adherente más débil en relaciones $\mathrm{B} 2 \mathrm{~B}$.

El § 308 (Klauselverbote mit Wertungsmöglichkeit) contempla un catálogo de "cláusulas prohibidas con posibilidad de valoración" ${ }^{20}$, conocida como la "lista gris", mientras que el § 309 (Klauselverbote ohne Wertungsmöglichkeit) establece un listado de "cláusulas prohibidas sin posibilidad de valoración" conocida como la "lista negra". Por su parte, el § 310 (Anwendungsbereich) regula el ámbito de aplicación de las normas anteriores, refiriéndose en su apartado 1 al contexto de relaciones entre empresarios.

En cuando a los conceptos de consumidor y empresario, se incorporaron en una reforma anterior, de fecha 27 de junio de 2000 (Gesetz über Fernab-

${ }^{18}$ Mato (2015), p. 252.

${ }^{19}$ Traducción de Vives (2002), p. 1246. Texto original: § 307 Inhaltskontrolle (1) Bestimmungen in Allgemeinen Geschäftsbedingungen sind unwirksam, wenn sie den Vertragspartner des Verwenders entgegen den Geboten von Treu und Glauben unangemessen benachteiligen. Eine unangemessene Benachteiligung kann sich auch daraus ergeben, dass die Bestimmung nicht klar und verständlich ist. (2) Eine unangemessene Benachteiligung ist im Zweifel anzunehmen, wenn eine Bestimmung 1. mit wesentlichen Grundgedanken der gesetzlichen Regelung, von der abgewichen wird, nicht zu vereinbaren ist oder 2. wesentliche Rechte oder Pflichten, die sich aus der Natur des Vertrags ergeben, so einschränkt, dass die Erreichung des Vertragszwecks gefährdet ist. (3) Die Absätze 1 und 2 sowie die $\S 3308$ und 309 gelten nur für Bestimmungen in Allgemeinen Geschäftsbedingungen, durch die von Rechtsvorschriften abweichende oder diese ergänzende Regelungen vereinbart werden. Andere Bestimmungen können nach Absatz 1 Satz 2 in Verbindung mit Absatz 1 Satz 1 unwirksam sein.

${ }^{20}$ Vives (2002), p. 1246.

${ }^{21}$ Ibid. 
satzverträge und andere Fragen des Verbraucherrechts sowie zur Umstellung von Vorschriften auf Euro). De acuerdo con lo anterior, la noción de consumidor (Verbraucher), dispuesta en el $\S 13^{22}$, corresponde a toda persona natural que celebra un negocio jurídico con una finalidad no atribuible preponderantemente a su actividad empresarial o profesional ${ }^{23}$. A su turno, empresario (Unternehmer), definido en el $\S 14^{24}$, es una persona natural o jurídica o una sociedad con capacidad jurídica que, al celebrar un negocio jurídico, actúa en el ejercicio de su oficio, negocio o profesión ${ }^{25}$.

Como es posible advertir, la expresión Unternehmer es bastante amplia y no restringida a pequeños-medianos empresarios. Sin embargo, cabe hacer una importante prevención. La extensión de la protección a empresarios es, normativa y comparativamente menor a la de los consumidores. En efecto, de acuerdo con el tenor del $\S 310$, no todos los supuestos contenidos en la lista gris de cláusulas abusivas resultan aplicables a contratos B2B y la lista negra del $\S 309$, se excluye por completo. No obstante, la jurisprudencia de todas formas ha utilizado estas normas para construir interpretaciones que conducen a la declaración de abusividad en relaciones entre empresarios ${ }^{26}$.

Como hemos sostenido en otro trabajo ${ }^{27}$, en el contexto descrito cobra especial relevancia el control de contenido del $\S 307$ como la principal norma que configura la protección al empresario más débil en relaciones $\mathrm{B}^{2} \mathrm{~B}^{28}$. A esto, cabe añadir, la tendencia jurisprudencial que interpreta de manera extensiva los $\S 308-309$, justificando este ejercicio en la llamada "eficacia indiciaria" (Indizwirkung) ${ }^{29}$ de ese listado de cláusulas abusivas. De todas formas, la jurisprudencia alemana ha realizado distinciones a la hora de aplicar estas interpretaciones, haciendo una diferenciación entre tipos de empresarios ajena al $\S 14$ del $B G B$. Así, por vía de interpretación y aplicación, aumenta la protección al pequeño empresario, matizando hasta excluir a las grandes empresas.

${ }^{22} \S 13$ Verbraucher. Verbraucher ist jede natürliche Person, die ein Rechtsgeschäft zu Zwecken abschließt, die überwiegend weder ihrer gewerblichen noch ihrer selbständigen beruflichen Tätigkeit zugerechnet werden können.

${ }^{23}$ Traducción libre.

${ }^{24} \S 14$ Unternehmer (1) Unternehmer ist eine natürliche oder juristische Person oder eine rechtsfähige Personengesellschaft, die bei Abschluss eines Rechtsgeschäfts in Ausübung ihrer gewerblichen oder selbständigen beruflichen Tätigkeit handelt (2) Eine rechtsfähige Personengesellschaft ist eine Personengesellschaft, die mit der Fähigkeit ausgestattet ist, Rechte zu erwerben und Verbindlichkeiten einzugehen.

${ }^{25}$ Traducción libre.

${ }^{26}$ Drygala (2012), p. 44.

${ }^{27}$ Morales, Mendoza y Munita (2020).

${ }^{28}$ Schmith \& Ulmer (2010), p. 36.

${ }^{29}$ Véanse sentencias en: Schmith \& Ulmer (2010), pp. 37-39; Mato (2015), pp. 258-261; Müller (2018), p. 155. 
III. EXTENSIÓN DEL CONTROL DE CLÁUSULAS ABUSIVAS A CONTRATOS B2B: CRÍTICAS Y PROPUESTAS DE REFORMA

La interpretación jurisprudencial explicada más atrás no ha tenido una plena $\operatorname{acogida}^{30}$.

Así, explica Beate Gsell, por tratarse de relaciones jurídicas distintas (B2C-B2B), la aplicación indirecta de los §§ 308-309 a través del § 307 es criticada por eliminar la especialidad de ellas mismas que, mediante una adecuada interpretación, resultan aplicables a relaciones de consumo ${ }^{31}$.

Por otro lado, el hecho de que la jurisprudencia haga una distinción en la práctica entre tipos de empresarios cuando la ley no distingue, también genera reproches ${ }^{32}$. Así, entre otros argumentos, recalcan Matthias Lehmann \& Johannes Ungerer que la protección del empresario "débil" frente al "fuerte" no es una labor que corresponda definir en la regulación de condiciones generales, sino al derecho de la competencia ${ }^{33}$.

Como consecuencia, el Ministerio Federal de Justicia y Protección al Consumidor alemán (Bundesministeriums der Justiz und für Verbraucherschutz) tomó nota del debate e impulsó la discusión encargando un proyecto de investigación que encabezó el Dr. Lars Leuschner, titulado "Términos y condiciones generales para contratos entre empresas: con especial consideración a las limitaciones de responsabilidad” (AGB-Recht für Verträge 212 zwischen Unternehmen Unter besonderer Berücksichtigung von Haftungsbeschränkungen), cuyo informe final fue presentado el 30 de septiembre de $2014^{34}$. En síntesis, el objetivo perseguido era determinar si se requiere o no una reforma en esta materia, labor que se llevó a cabo a través del análisis de la jurisprudencia del Tribunal Federal de Justicia de Alemania ${ }^{35}$, análisis de las limitaciones de responsabilidad habituales dentro del sector industrial ${ }^{36}$ y revisión de derecho comparado ${ }^{37}$. Destaca también en la metodología un apartado de investigación empírica, realizada a través de cuestionarios aplicados a expertos en arbitraje y trabajadores de empresas ${ }^{38}$. Asimismo, el estudio reafirmó que, a diferencia de otros ordenamientos, las empresas en Alemania no pueden limitar su responsabilidad en contratos masivos estandarizados. Se explica que esto es así por la jurisprudencia, que es muy estricta frente a los acuerdos individuales, realizando un estricto

${ }^{30}$ Debate que comienza a tomar fuerza como consecuencia de la elaboración del DCFR en Europa. Micklitz (2013), p. 287.

${ }^{31}$ Gsell (2017), p. 246.

${ }^{32}$ Lehmann \& Ungerer (2017), pp. 306-311.

${ }^{33}$ Op. cit., pp. 307-308.

${ }^{34}$ Leuschner (2014).

${ }^{35}$ Op. cit., pp. 15-41.

${ }^{36}$ Op. cit., pp. 43-90.

${ }^{37}$ Op. cit., pp. 91-136.

${ }^{38}$ Op. cit., pp. 137-285. 
control de contenido en las restricciones (e, incluso, en las extensiones) de responsabilidad ${ }^{39}$.

En dicho sentido, sostiene Lars Leuschner que la ley debe representar un importante papel, por lo que se sugiere realizar una reforma legal eliminando la "fórmula de previsibilidad" (Vorhersehbarkeitsformel) utilizada por la jurisprudencia, mediante la cual la responsabilidad por el incumplimiento de obligaciones contractuales esenciales debe incluir al menos los daños previsibles y típicos del contrato ${ }^{40}$, introduciendo expresamente la admisibilidad de restricciones de responsabilidad general entre empresarios, haciendo primar la voluntad de las partes $^{41}$. También propone la existencia de una cifra límite y determinante de control de cláusulas en relaciones entre empresarios, evitando así problemas de delimitación ${ }^{42}$. A su juicio, esto no es óbice para introducir una disposición según la cual el control de cláusulas pueda ser aplicable en otros casos por sobre esa cifra límite, si hubiera un desequilibrio económico considerable.

Asimismo, han habido algunas iniciativas de reforma al $B G B$ en los $\S \S 305$ y $310^{43}$. Por su parte, el propio Lars Leuschner, publicó en 2015 su propuesta de reforma siguiendo la línea de su informe de 2014 (Reformvorschläge für die AGB-Kontrolle im unternehmerischen Rechtsverkehr) ${ }^{44}$. Solo por mencionar otro ejemplo, bajo el alero de asociaciones empresariales, se crea la "Propuesta legislativa de modificación de la regulación de los términos y condiciones generales en las transacciones comerciales" (Gesetzgebungsvorschlag zur Änderung des AGB-Rechts im unternehmerischen Geschäftsverkehr ${ }^{45}$. Esta propuesta, así como sus versiones anteriores que datan desde 2008, recuerdan que el espíritu original era otorgar mayor elasticidad a las relaciones entre empresarios, no siendo equiparadas a las relaciones $\mathrm{B}^{2} \mathrm{C}^{46}$, así, sugieren cambios concretos a la redacción de los $\S \S 305,307$ y 310. Como contrapartida, existe también un movimiento llamado "pro AGB- Recht" que está conformado por más de treinta microempresarios, los cuales abogan por mantener la protección de las empresas más débiles sin alterar el status quo, destacando las ventajas que legislación alemana les otorga, en comparación a otros sistemas ${ }^{47}$.

${ }^{39}$ Leuschner (2014), p. 10.

${ }^{40}$ Op. cit., pp. 10, 28-31, 289-290.

${ }^{41}$ Op. cit., pp. 10, 290-291.

${ }^{42}$ Op. cit., pp. 10, 28-31, 289-290.

${ }^{43}$ Véase síntesis de otras propuestas en Leuschner (2014), p. 12 y en MÜLler (2018), pp. 157159. Un estado actual de la discusión se puede revisar en Herresthal (2020).

${ }^{44}$ Leuschner (2015a), pp. 1045-1052. Propuesta que fue criticada por Westphalen (2015), pp. 1316-1325, quien, a su vez, plantea una propia.. En el mismo número, replica LEUSCHNER (2015b), pp. 1326-1332.

${ }^{45}$ UUltima versión de junio de 2018. Disponible en www.zvei.org/fileadmin/user_upload/ Themen/Maerkte_Recht/Allgemeine_Geschaeftsbedingungen_im_unternehmerischen_ Geschaeftsverkehr/pdf/AGB-Initiative-Positionspapier-Stand-06-2018.pdf [fecha de consulta: 11 de febrero de 2019].

${ }^{46}$ Recalca la misma idea Müller (2018), p. 153.

${ }^{47}$ Así lo explican en su propia página web www.pro-agb-recht.de [fecha de consulta: 5 de febrero de 2019]. 


\section{BibliografíA CITADA}

Albiez, Klaus Jochen (2002). "Un nuevo derecho de obligaciones. La reforma 2002 del BGB”. Anuario de Derecho Civil, vol. 55, n. ${ }^{\circ}$ 3. Madrid.

Drygala, Tim (2012). Unfair contract terms in business to business transaction, in Tim Drygala, Bettina Heiderhoff, Marco Staake, Grzegorz Zmij (eds.). Private Autonomy in Germany and Poland and in the Common European Sales Law. Munich: Sellier European Law Publishers.

GsELL, Beate (2017). "Deutsche Erfahrungen mit der begrenzten Erstreckung der Klauselkontrolle auf den unternehmerischen Verkehr”, in Johann KInDL, María Pilar Perales \& Tatiana Arroyo Vendrell (eds.). Standardisierte Verträge-zwischen Privatautonomie und rechtlicher Kontrolle, Baden-Baden: Nomos.

Herresthal, Carsten (2020). Reform der AGB-Kontrolle im B2B-Bereich. BadenBaden: Nomos.

Lehmann, Matthias \& Johannes Ungerer (2017). "Differenzierte Unternehmerbegriffe und differenzierte Missbräuchlichkeitsanforderungen innerhalb der B2B-Inhaltskontrolle", in Johann Kindl, María Pilar Perales Viscasillas \& Tatiana Arroyo Vendrell (eds.). Standardisierte Verträge-zwischen Privatautonomie und rechtlicher Kontrolle, Baden-Baden: Nomos.

LEUSCHNER, Lars (2014). “AGB-Recht für Verträge zwischen Unternehmen“ Unter besonderer Berücksichtigung von Haftungsbeschränkungen”. Forschungsprojekt im Auftrag des Bundesministeriums der Justiz und für Verbraucherschutz. Disponible en www.bmjv.de/SharedDocs/Downloads/DE/Fachinformationen/ Abschlussbericht-AGB-Forschungsprojekt.pdf?_blob=publicationFile [fecha de consulta: 6 de febrero de 2019].

Leuschner, Lars (2015a). "Reformvorschläge für die AGB-Kontrolle im unternehmerischen Rechtsverkehr". ZIP-Zeitschrift für Wirtschaftsrecht, vol. 36, n 22. Köln.

Leuschner, Lars (2015b). "Noch einmal: Reformvorschläge für die AGB-Kontrolle im unternehmerischen Rechtsverkehr". ZIP-Zeitschrift fü $r$ Wirtschaftsrecht, vol. 36, $n^{0} 28$. Köln.

Mato, María Natalia (2015). "El control de contenido en la contratación mediante condiciones generales entre empresarios en el Derecho Comparado y Europeo". Cuadernos de Derecho Transnacional, vol. 7, n. ${ }^{\circ}$ 2. Getafe, Madrid.

Micklitz, Hans-Wolfgang (2013). "Do Consumers and Businesses Need a New Architecture of Consumer Law? A Thought Provoking Impulse". Yearbook of European Law, vol. 32, issue 1. Oxford.

Momberg Uribe, Rodrigo (2016). “Análisis de los modelos de vinculación del código civil y la legislación de protección al consumidor: hacia un principio general de protección de la parte débil en el Derecho Privado". Revista Chilena de Derecho, vol. 43, n. ${ }^{\circ}$ 2. Santiago.

Morales, María Elisa, Pamela Mendoza y Renzo Munita (2020). "Cláusulas abusivas entre empresas. Evolución en los principales sistemas de Derecho Comparado”. Vniversitas, vol. 69, n. ${ }^{\circ}$ 141. Bogotá: Inédito, aceptado. 
MÜLLER, Werner (2018). "AGB-Kontrolle auf dem Prüfstand". IWRZ- Zeitschrift für Internationales Wirtschaftsrecht, $\mathrm{n}^{\circ} 4$. Baden-Baden.

Sснмiтн, Christoph \& Detlef Ulmer (2010). Allgemeine Geschäftsbedingungen und Verträge für Unternehmen: Chancen und Risiken. Berlin: Springer.

Vives, María Luisa (2002). "Traducción de la reforma 2002 del BGB”. Anuario de Derecho Civil, vol. 55, n. ${ }^{\circ}$ 3. Madrid.

WACKE, Andreas (2013). "Las reformas más importantes del BGB desde su promulgación en 1900, con especial referencia al derecho de obligaciones". Revista Chilena de Derecho, vol. 40, n. ${ }^{\circ}$ 2. Santiago.

WestPhalen, Friedrich Graf von (2015). "Schwellenwert und Neuregelung einer vertraglichen Haftungsbegrenzung - ist das der „Königsweg“ einer Reform des AGB-Rechts?”. ZIP-Zeitschrift für Wirtschaftsrecht, vol. 36, n 28. Köln.

Zimmermann, Reinhard (2005) "Consumer Contract Law and General Contract Law: The German Experience". Current Legal Problems, vol. 58, issue 1. Oxford.

Zweigert, Konrad \& Hein KöTz (1998), An Introduction to Comparative Law. (trad.) Tony Weir. 3rd ed. Oxford: Oxford University Press.

\section{Normas citadas}

"Bürgerliches Gesetzbuch (BGB)". Reichsgesetzblatt. No 21, 18. August 1896.

Directiva 93/13/CEE del Consejo, de 5 de abril de 1993, sobre las cláusulas abusivas en los contratos celebrados con consumidores. DOUE. L95 de 21 de abril de 1993.

"Gesetz zur Regelung des Rechts der Allgemeinen Geschäftsbedingungen (AGBG)". Bundesgesetzblatt. No 141, 9. Dezember 1976.

"Gesetz zur Änderung des AGB-Gesetzes und der Insolvenzordnung". Bundesgesetzblatt. No 36, 19. Juli 1996.

"Gesetz über Fernabsatzverträge und andere Fragen des Verbraucherrechts sowie zur Umstellung von Vorschriften auf Euro". Bundesgesetzblatt. No 28 , 27 . Juni 2000.

"Gesetz zur Modernisierung des Schuldrechts". Bundesgesetzblatt. No 61, 26. November 2001.

SigLA Y ABREVIATURAS

AGB Allgemeine Geschäftsbedingungen

AGBG Gesetz zur Regelung des Rechts der Allgemeinen Geschäftsbedingungen

B2B Business to business

B2C Business to consumer

$B G B$ Bürgerliches Gesetzbuch 


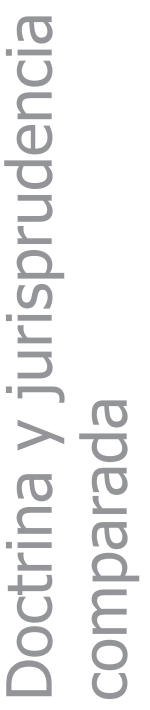

\author{
CEE Comunidad Económica Europea \\ DCFR Draft Common Frame of Reference \\ Dr. doctor \\ DOUE Diario Oficial de la Unión Europea \\ eds. editores \\ Fondecyt Fondo Nacional de Desarrollo Cien- \\ tífico y Tecnológico \\ Ibid. Ibidem \\ n. ${ }^{\circ}$ a veces $\mathrm{N}^{\circ}$ número \\ op. cit. opere citato (obra citada) \\ p. página \\ pp. páginas \\ vol. volumen \\ www. World Wide Web
}

